\title{
Percutaneous coronary intervention in patients with multi-vessel coronary artery disease: a focus on physiology
}

\author{
Yun-Kyeong Cho and Chang-Wook Nam
}

Division of Cardiology, Department of Internal Medicine, Keimyung University Dongsan Medical Center, Daegu, Korea

Received: January 5, 2018

Accepted: January 16, 2018

\section{Correspondence to}

Chang-Wook Nam, M.D.

Division of Cardiology, De-

partment of Internal Medicine,

Keimyung University Dongsan

Medical Center, 56 Dalseong-ro,

Jung-gu, Daegu 41931, Korea

Tel: $+82-53-250-8015$

Fax: $+82-53-250-7034$

E-mail: ncwcv@dsmc.or.kr
Multi-vessel coronary artery disease (MVD) frequently features ambiguous or intermediate lesions that may be both serial and complex, suggesting that multiple regions require revascularization. Percutaneous coronary intervention (PCI) is associated with various challenges such as appropriate identification of lesions that should be treated, the choice of an optimum revascularization method, and limitations of long-term outcomes. Optimal patient selection and careful targeting of lesions are key when planning treatment. Physiology-guided decision-making (based on the fractional flow reserve) can overcome the current limitations of PCI used to treat MVD regardless of clinical presentation or disease subtype, as confirmed in recent clinical trials. Here, we review the use of physiology-guided PCI for patients with MVD, and their early and late outcomes.

Keywords: Coronary artery disease; Fractional flow reserve; Prognosis

\section{INTRODUCTION}

Multi-vessel coronary artery disease (MVD) is frequently observed in both stable and unstable patients; the prevalence of the condition ranges from $30 \%$ to $60 \%[1,2]$. The risk of death increases as more major epicardial coronary arteries become involved [3]. Coronary revascularization is associated with perioperative or periprocedural complications and risks of major adverse cardiac events (MACEs). Therefore, the chosen revascularization strategy must be appropriate. As many angiographically significant lesions are not in fact hemodynamically significant [4], the fractional flow reserve (FFR), which reflects the functional significance of a particular coronary lesion, affects treatment decisions and clinical outcomes. Here, we review anatomically and physiologically guided percutaneous coronary intervention (PCI) in patients with MVD, and their short- and long-term outcomes.

\section{REVASCULARIZATION IN THE ABSENCE OF PHYSIOLOGICAL GUIDANCE}

Although PCI techniques and devices have remarkably improved in recent years, coronary artery bypass surgery (CABG) remains the gold standard treatment for MVD patients $[5,6]$. Several clinical trials comparing surgical and percutaneous techniques have revealed the superiority of CABG. The BARI (Bypass Angioplasty Revascularization Investigation) trial evaluated the use of CABG combined with angioplasty in MVD patients [7]. At the 10-year follow-up, angioplasty alone afforded a survival rate similar to that of the combined treatment (71.0\% vs. 
$73.5 \%, p=0.18)$. However, the angioplasty group experienced a substantially higher rate of later revascularization than the CABG group (76.8\% vs. $20.3 \%, p<0.001$ ) [8].

The ARTS (Arterial Revascularization Therapies Study) trial compared the clinical outcomes of MVD patients treated via bare metal stenting rather than CABG [9]. At the 5-year follow-up, the frequency of repeat revascularization was significantly higher in those who underwent stenting compared to CABG (30.3\% vs. $8.8 \%, p<0.001$ ). Thus, the composite event-free survival rate after CABG was better than that after stenting (78.2\% vs. $58.3 \%, p<$ $0.0001)[10]$.

The SYNergy between percutaneous coronary intervention with TAXus and cardiac surgery (SYNTAX) trial compared CABG and PCI (individually, not combined) followed by paclitaxel-eluting stent placement in patients with 3 vessel disease (3VD) or left main disease, or both [11]. The SYNTAX score (SS), a novel measure based on the anatomy of lesions causing stenosis $>50 \%$ of vessels of diameter $>1.5 \mathrm{~mm}$, was used to define the extent, complexity, and severity of coronary artery disease and to predict outcomes. In patients with $3 \mathrm{VD}$, the frequencies of major adverse cardiac and cerebrovascular events (MACCEs) including death, myocardial infarction (MI), and a need for repeat revascularization at the 5-year follow-up, increased over time, as did the stroke rate $37.5 \%$ in PCI vs. $24.2 \%$ in CABG patients, $p<0.001$ ) [12]. Similar proportions of low-SS $(\mathrm{SS} \leq 22)$ patients treated via either CABG or PCI attained the primary endpoints, but the intermediate- (23 to 32 ) and high-SS ( $\geq 33$ ) groups exhibited significantly increased MACCE rates after PCI compared to CABG.

In the recent Bypass Surgery and Everolimus-Eluting Stent Implantation in the Treatment of Patients with Multivessel Coronary Artery Disease (BEST) trial, although PCI with placement of everolimus-eluting stents afforded clinical outcomes that were not inferior to CABG at 2 years, death, MI, and the need for target-vessel revascularization during long-term follow-up (median, 4.6 years) were more frequent in the PCI group (15.3\% vs. $10.6 \%$ in the CABG group, $p=0.04$ ) [13].

CABG affords the advantages of excellent long-term patency (particularly when the left internal thoracic or mammary artery serves as a conduit) and wide applicability even when the anatomy is complex. Despite recent technical improvements in coronary stents, PCI is more frequently associated with incomplete revascularization (IR) than CABG, increasing the risk for adverse events. In addition, the risk for procedure-related strokes during CABG (which is higher than that during PCI) is falling because off-pump CABG avoids any excessive manipulation of the aorta, reducing the stroke risk. The difference in approach to the lesion may explain the superiority of CABG [14]. PCI treatment targets severely diseased coronary arteries but CABG less seriously affected (or indeed near-normal) coronary arteries. Thus, the long-term outcomes of either type of revascularization may differ. Therefore, appropriate selection of the PCI target is extremely important in MVD patients to prevent adverse events associated with inadequate stenting. Although many clinical trials have evaluated the efficacy and safety of various revascularization methods for MVD patients (Table 1), many issues remain and additional trials are necessary.

\section{PHYSIOLOGY-GUIDED REVASCULARIZATION- OF MVD PATIENTS}

\section{Fractional flow reserve-guided $\mathrm{PCI}$ to treat MVD}

It is generally accepted that revascularization of coronary lesions that exhibit reversible ischemia can relieve angina symptoms and improve patient outcomes. However, coronary angiography has certain limitations when used to identify ischemia-producing stenoses. The extent of anatomical stenosis of intermediate lesions often does not reflect functional severity [15]. It can be difficult to identify lesions causing ischemia in MVD patients. Although noninvasive stress imaging modalities can be useful, they have certain limitations when used in efforts to accurately identify ischemia-producing lesions [16]. By contrast, the FFR is a lesion-specific index measured using a pressure wire that rapidly determines functional significance by calculating the ratio of the distal coronary pressure to the aortic pressure that is simultaneously measured using a guiding catheter. An $\mathrm{FFR} \leq 0.80$ indicates myocardial ischemia with an accuracy $>90 \%$. Therefore, the FFR should be used to guide coronary revascularization [6]. The Fractional Flow Reserve Versus Angiography for Multivessel Evaluation (FAME) trial compared angiography-guided PCI with FFR-guided PCI in MVD patients [17]. The 1-year rate of 
Table 1. Published studies on revascularization strategies

\begin{tabular}{|c|c|c|c|c|c|}
\hline Study & Study design & $\begin{array}{l}\text { No. of } \\
\text { patients }\end{array}$ & $\begin{array}{l}\text { Comparison } \\
\text { arms }\end{array}$ & Inclusion criteria & Outcomes \\
\hline BARI $[7,8]$ & Randomized & 1,829 & $\begin{array}{l}\text { PTCA vs. } \\
\text { CABG }\end{array}$ & $\begin{array}{l}\text { Angiographically documented } \\
\text { MVD with clinically severe } \\
\text { angina or objective evidence } \\
\text { of ischemia requiring } \\
\text { revascularization }\end{array}$ & $\begin{array}{l}\text { 5-Year survival: } 86.3 \% \text { for PTCA } \\
\text { vs. } 89.3 \% \text { for CABG }(p=0.19) \\
\text { 10-Year survival: } 71.0 \% \text { for PTCA } \\
\text { vs. } 73.5 \% \text { for CABG }(p=0.18)\end{array}$ \\
\hline $\operatorname{ARTS}[9,10]$ & Randomized & 1,205 & $\begin{array}{l}\text { PCI with BMS } \\
\text { vs. CABG }\end{array}$ & $\begin{array}{l}\text { Stable angina pectoris, unsta- } \\
\text { ble angina or silent ischemia } \\
\text { \& at least } 2 \text { new lesions that } \\
\text { were located in different } \\
\text { vessels and territories (not } \\
\text { including the LMCA) }\end{array}$ & $\begin{array}{l}\text { MACCE-free survival at } 1 \text { year: } \\
73.8 \% \text { for PCI vs. } 87.8 \text { for CABG } \\
(p<0.001) \\
\text { Event-free survival at } 5 \text { years: } \\
58.3 \% \text { for PCI vs. } 78.2 \% \text { for CABG } \\
(p<0.0001)\end{array}$ \\
\hline $\begin{array}{l}\text { SYNTAX } \\
{[11,12]}\end{array}$ & Randomized & $\begin{array}{l}1,800 \\
(1,095 \\
\text { in } 3 \mathrm{VD} \\
705 \text { in } \\
\text { LMCA) }\end{array}$ & $\begin{array}{l}\text { PCI with PES } \\
\text { vs. CABG }\end{array}$ & $\begin{array}{l}3 \text { Vessel disease and LMCA } \\
\text { disease (alone or with } \mathrm{VD} \text {, } \\
2 \mathrm{VD} \text {, or } 3 \mathrm{VD} \text { ) }\end{array}$ & $\begin{array}{l}\text { 12-Month MACCE in all: } 17.8 \% \\
\text { for PCI vs. } 12.4 \% \text { for CABG }(p= \\
\text { 0.002) } \\
\text { 12-Month MACCE in } 3 \text { VD: } \\
\text { 19.2\% for PCI vs. } 11.5 \% \text { for CABG } \\
(p<0.001) \\
\text { 5-Year MACCE in all: } 37.3 \% \text { for PCI } \\
\text { vs. } 26.9 \% \text { for CABG }(p<0.001) \\
\text { 5-Year MACCE in } 3 \text { VD: } 37.5 \% \text { for } \\
\text { PCI vs. } 24.2 \% \text { for CABG }(p<0.001)\end{array}$ \\
\hline $\operatorname{BEST}[13]$ & Randomized & 880 & $\begin{array}{l}\text { PCI with EES } \\
\text { vs. CABG }\end{array}$ & $\begin{array}{l}\text { Angiographically confirmed } \\
\text { MVD with stenoses of more } \\
\text { than 70\% in major epicardial } \\
\text { vessels in the territories of at } \\
\text { least two coronary arteries }\end{array}$ & $\begin{array}{l}\text { MACE at } 2 \text { years: } 11.0 \% \text { for PCI vs. } \\
7.9 \% \text { for CABG }(p=0.32) \\
\text { MACE at } 4.6 \text { years: } 15.3 \% \text { for PCI } \\
\text { vs. } 10.6 \% \text { for CABG }(p=0.04)\end{array}$ \\
\hline FAME [17-19] & Randomized & 1,005 & $\begin{array}{l}\text { FFR-guid- } \\
\text { ed PCI vs. } \\
\text { angiogra- } \\
\text { phy-guided } \\
\text { PCI }\end{array}$ & $\begin{array}{l}\text { MVD which lesions had } \\
\text { stenoses of at least } 50 \% \text { of } \\
\text { their diameter }\end{array}$ & $\begin{array}{l}\text { 1-Year MACE: } 13.2 \% \text { for FFR } 18.3 \% \\
\text { for angiography }(p=0.02) \\
\text { 2-Year MACE: } 17.9 \% \text { for FFR } \\
22.4 \% \text { for angiography }(p=0.08) \text {, } \\
\text { 5-Year MACE: } 28.0 \% \text { for FFR } \\
\text { 31.0\% for angiography }(p=0.31)\end{array}$ \\
\hline FSS [21] & Prospective & 497 & SS vs. FSS & $\begin{array}{l}\text { FFR guided group in } \\
\text { the FAME }\end{array}$ & $\begin{array}{l}\text { 1-Year MACE: } 8.4 \%, 10.2 \%, \text { and } \\
20.9 \% \text { in the low-, medium-, and } \\
\text { high-SS groups vs. } 9.0 \%, 11.3 \% \text {, } \\
\text { and } 26.7 \% \text { in the low-, medium- } \\
\text { and high-FSS groups (Harrell's C } \\
\text { of the FSS, } 0.677 \text { vs. SS, o.630; } \\
p=0.02 \text { ) }\end{array}$ \\
\hline $\begin{array}{l}\text { DANAMI-3- } \\
\text { PRIMULTI } \\
{[30]}\end{array}$ & Randomized & 627 & $\begin{array}{l}\text { No further } \\
\text { invasive } \\
\text { treatment vs. } \\
\text { FFR-guided } \\
\text { CR before } \\
\text { discharge }\end{array}$ & $\begin{array}{l}\text { STEMI patients with an } \\
\text { angiographic diameter } \\
\text { stenosis of greater than } 50 \% \\
\text { in one or more non-IRA after } \\
\text { a PCI of an IRA }\end{array}$ & $\begin{array}{l}\text { MACE in the non-IRA group at } 27 \\
\text { months: } 13 \% \text { for FFR-guided CR } \\
\text { vs. } 22 \% \text { for no further invasive } \\
\text { treatment }(p=0.004)\end{array}$ \\
\hline
\end{tabular}


Table 1. Continued

\begin{tabular}{|c|c|c|c|c|c|}
\hline Study & Study design & $\begin{array}{c}\text { No. of } \\
\text { patients }\end{array}$ & Comparison arms & Inclusion criteria & Outcomes \\
\hline $\begin{array}{l}\text { Compare- } \\
\text { Acute [31] }\end{array}$ & Randomized & 885 & $\begin{array}{l}\text { FFR-guided } \\
\text { CR vs. no } \\
\text { revascularization } \\
\text { of non-IRA }\end{array}$ & $\begin{array}{l}\text { STEMI and MVD patients } \\
\text { who had a non-IRA with a } \\
\text { stenosis of } 50 \% \text { or greater after } \\
\text { the primary PCI of an IRA }\end{array}$ & $\begin{array}{l}\text { 12-Month MACCE: } 7.8 \% \text { for } \\
\text { FFR-guided CR vs. } 20.5 \% \\
\text { for no revascularization } \\
(p<0.001)\end{array}$ \\
\hline FRAME-AMI & Randomized & 1,292 & $\begin{array}{l}\text { FFR-guided } \\
\text { strategy vs. } \\
\text { angiography- } \\
\text { guided strategy }\end{array}$ & $\begin{array}{l}\text { AMI and MVD patients who } \\
\text { had a non-IRA stenosis with } \\
>50 \% \text { stenosis after a PCI of } \\
\text { an IRA }\end{array}$ & Recruiting \\
\hline
\end{tabular}

BARI, Bypass Angioplasty Revascularization Investigation; PTCA, percutaneous transluminal coronary balloon angioplasty; CABG, coronary artery bypass grafting; MVD, multivessel disease; ARTS, Arterial Revascularization Therapies Study; PCI, percutaneous coronary intervention; BMS, bare metal stent; LMCA, left main coronary artery; MACCE, major adverse cardiac and cerebrovascular event; SYNTAX, SYNergy between percutaneous coronary intervention with TAXus and cardiac surgery; 3VD, 3 vessel disease; PES, paclitaxel-eluting stent; BEST, Bypass Surgery and Everolimus-Eluting Stent Implantation in the Treatment of Patients with Multivessel Coronary Artery Disease; EES, everolimus-eluting stent; MACE, major adverse cardiac events; FAME, Fractional Flow Reserve Versus Angiography for Multivessel Evaluation; FFR, fractional flow reserve; FSS, functional SYNTAX score; SS, SYNTAX score; DANAMI-3-PRIMULTI, Primary PCI in Patients With ST-elevation Myocardial Infarction and Multivessel Disease: Treatment of Culprit Lesion Only or Complete Revascularization; CR, complete revascularization; STEMI, ST segment elevation myocardial infarction; IRA, infarct-related artery; FRAME-AMI, FFR versus Angiography-Guided Strategy for Management of AMI with Multivessel Disease.

MACE including death, nonfatal MI, and repeat revascularization was significantly higher in the angiographic than FFR group (combined, $18.3 \%$ vs. $13.2 \%, p=0.02$; death, $1.8 \%$ vs. $3.0 \%, p=0.19$; MI, $5.7 \%$ vs. $8.7 \%, p=0.07$; repeat revascularization, $6.5 \%$ vs. $9.5 \%, p=0.08)$. In addition, routine FFR significantly reduced both the mortality and MI rate at 2 years (combined, $8.4 \%$ vs. $12.9 \%, p=$ o.02) [18] and 5 years ( $28 \%$ vs. $31 \%, p=0.31)$, with no need for revascularization. However, the differences were not significant because of the small number of patients [19]. The FAME trial showed that FFR guidance was associated with a significantly lower incidence of MACE compared to angiographic guidance, without any significant increase in procedural time and a reduction in cost. In addition, it was found that routine FFR may minimize the need for PCI caused by an oculostenotic reflex. Subanalysis revealed that FFR-guided PCI in MVD patients was less costly than angiography-guided PCI and improved the quality of adjusted life-years measured 1 year later. Therefore, FFR-guided PCI is a rare example of a new technique that not only improves outcomes and patient quality-of-life, but also reduces costs [20]. However, further trials are necessary to validate the routine use of FFR in patients undergoing MV PCI.

\section{A functional SS for MVD patients}

Although the SS allows risk stratification and predicts the prognosis of MVD, it has the innate limitation of being angiographically based. Thus, the functional importance of a lesion may be underestimated or overestimated. Underestimation is associated with IR and an increased risk of adverse events. Overestimation reflects PCI of a functionally insignificant lesion, either affording no benefit or actually causing harm because revascularization is inadequate. To overcome these limitations, an additional functional evaluation (with an anatomical assessment) would help guide appropriate decision-making.

The functional SS (FSS) is recalculated SS that only evaluates ischemia-producing lesions as revealed by FFR. When applying the SS based on angiography to patients enrolled in the FAME trial, 32\% of the higher risk SS group moved to the lower risk group. MACE developed in $8.4 \%, 10.2 \%$, and $20.9 \%$ of the low-, medium-, and high-SS groups $(p=0.001)$; and in $9.0 \%, 11.3 \%$, and $26.7 \%$ of the low-, medium-, and high-FSS groups, respectively $(p<0.001)$. Further, the FSS better predicted the 1-year clinical outcomes than the SS (Harrell's C-values for the FSS, 0.677 vs. 0.630 for the SS, $p=0.02$ ) [21]. 


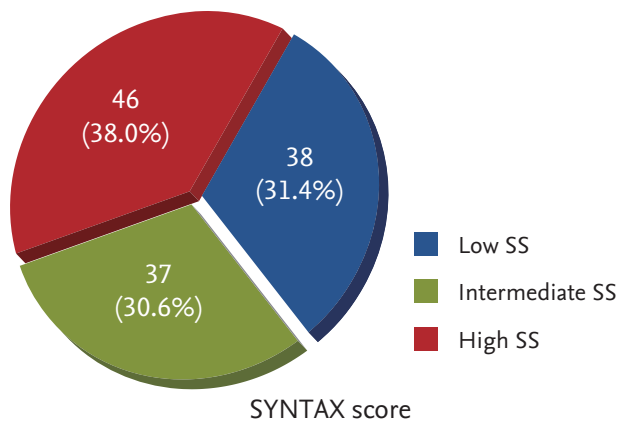

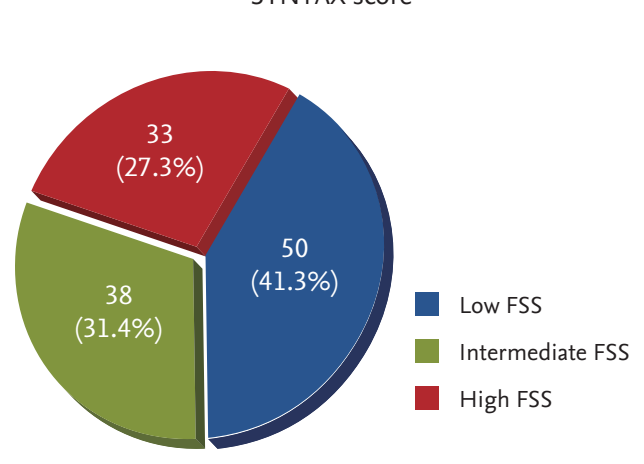

Functional SYNTAX score

Functional SYNTAX score

\section{A}

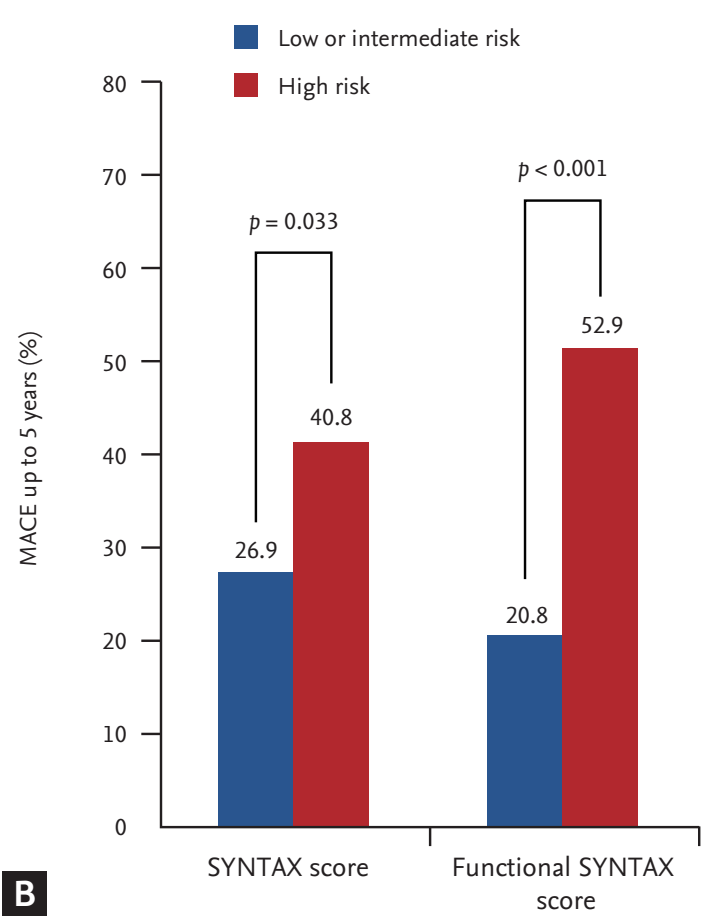

Figure 1. Subgrouping of study populations. (A) The proportions of the study population in terms of tertiles of the classic SYNTAX score (SS) and the functional SS (FSS). A total of $23.9 \%$ of patients in the high-SS tertile moved to the intermediate-FSS tertile and $4.4 \%$ to the low-FSS tertile. Further, $27.0 \%$ of patients in the intermediate-SS tertile moved to the low-FSS tertile. (B) The major adverse cardiac event (MACE) rates to 5 years. The difference in MACE frequency was greater between the low- and intermediate-risk FSS groups, and the high-risk SS group, than that between the low- and intermediate-risk SS groups, and the high-risk SS group. SYNTAX, SYNergy between percutaneous coronary intervention with TAXus and cardiac surgery.

The benefits afforded by the FSS are 2-fold. First, some patients at anatomically higher risk for MVD may be reclassified as being physiologically at lower risk, and thus can be appropriately treated via PCI. Second, physiology-based FSS is better than anatomy-based classic SS in terms of predicting outcomes after revascularization. Patients who moved from an anatomically higher risk group to a functionally lower risk group experienced outcomes similar to those of the anatomically lower risk group. However, patients who remained in a higher functional risk group experienced very poor outcomes after PCI, and thus should undergo a different form of revascularization such as CABG [22].

The FSS predicts not only short- but also long-term outcomes. We included 121 consecutive patients with MVD whose details are on the SYNTAX website, and calculated their SSs. The FFRs of angiographically ambiguous lesions were measured. FSSs were calculated by adding the scores of lesions with FFRs $\leq 0.80$ and ignoring lesions with FFRs $>0.80$. All of the patients were divided into MACE risk tertiles based on the SS, and MACE was followed up for 5 years. The SS range, mean \pm standard deviation, and median were 3 to $34,16.3$ \pm 6.8 , and 16.0, respectively. The figures for the FSS were o to $34,12.3 \pm 8.1$, and 11.0, respectively. The mean SS values were $8.6 \pm 2.6,15.6 \pm 1.7$, and $23.2 \pm 4.2$ in the low-, medium-, and high-SS groups, respectively $(p<0.001)$; whereas the mean FSS values were 5.0 $\pm 3.2,12.7 \pm 3.2$, and $23.0 \pm 4.1$ in the low-, medium-, and high-FSS groups $(p<$ 0.001 ). After determination of FSSs for all of the patients, $19.0 \%$ moved to a lower risk group. During 5 years of follow-up, the incidence of MACE in the high-SS group was significantly greater than that in the low- or intermediate-SS groups $(26.9 \%$ vs. $40.8 \%, p=0.033)$, as was also true of the FSS groups ( $20.8 \%$ vs. $52.9 \%, p<0.001$ ) (Fig. 1). Logistic regression analyses revealed that the FFS independently predicted long-term MACE (hazard ratio [HR], 3.025; 95\% confidence interval [CI], 1.408 to 
6.502; $p=0.005$ ). The benefits afforded by the FSS reflect not only physiological assessment based on the FFR, but also careful evaluation of anatomical complexity (SS calculations). Therefore, a combination of anatomical and physiological evaluations overcome the limitations of each method, identifying appropriate treatment plans and predicting prognosis. However, the FSS does not reflect clinical complexity. Addition of clinical risk factors to the FSS will improve predictability.

\section{Functionally complete revascularization in MVD patients}

IR is a surrogate marker of higher coronary complexity, greater clinical comorbidity, and more adverse outcomes after CABG or PCI to treat MVD. IR occurs more frequently after PCI, but is not rare after CABG. The effects of residual coronary stenosis after PCI or CABG vary; there is no universal definition of IR. In previous studies, IR was defined by reference to the severity of stenosis (e.g., $50 \%$ vs. $70 \%$ ) or by reference to the diameter of the vessel requiring treatment (e.g., 1.5 to $2.5 \mathrm{~mm}$ ). The residual SS (rSS) formally quantifies the extent and complexity of residual stenosis and can be calculated based on the level of obstructive coronary disease evident after PCI. When the rSS was assessed in patients of the ACUITY (Acute Catheterization and Urgent Intervention Triage strategY) trial, the measure was a strong independent predictor of ischemic outcomes and an rSS $>8$ was associated with a poorer prognosis [23].

The residual functional SS (rFSS) is defined as the rSS measured only in vessels with FFRs $\leq 0.8$ and is more discriminatory than anatomical or physiological assessment alone. When 385 patients who underwent $3 \mathrm{~V}$ FFR measurements after stent implantation were followed up for 2 years, the rFSS was significantly associated with MACE (HR, 1.09; 95\% CI, 1.02 to 1.17; $p=0.018$ ). When clinical risk factors were added, the ability of the rFSS to predict MACE increased more (by 3.5\%, $p=0.002$ ) than the predictive abilities of the FSS, rSS, and rFSS [24]. Analysis of pooled data from the SYNTAX, PRECOMBAT (Premier of Randomized Comparison of Bypass Surgery versus Angioplasty Using Sirolimus-Eluting Stent in Patients with Left Main Coronary Artery Disease), and BEST trials revealed that PCI that achieved complete revascularization (CR) afforded a long-term survival duration similar to that after CABG with CR.
However, PCI associated with IR was associated with a poor prognosis [25]. Although angiographic CR is associated with improved outcomes, CR is not always possible in MVD patients undergoing PCI. As treatment of angiographically significant but functionally insignificant lesions can be safely deferred, selective coronary revascularization guided by FFR measurements should be considered [26]. Therefore, when treating MVD, the treatment strategy chosen should seek to achieve functionally CR.

\section{Physiology-guided $\mathrm{PCl}$ in patients with acute $\mathrm{MI}$ and MVD}

The use of FFR to assess the hemodynamic significance of nonculprit coronary artery lesions during the acute phase of MI enhances risk stratification and shortens hospital stay by reducing the need for additional tests detecting residual myocardial ischemia. If coronary microvascular dysfunction is present, maximal hyperemia may not be readily attained during FFR measurements; indeed, the FFR has not often been used to evaluate patients with acute MI, because the maximum attainable flow is less and the FFR gradient smaller in such patients. In other words, the FFR is not a reliable assessment of the severity of possibly culprit lesions under such circumstances. Only a few studies have evaluated the FFRs of infarct-related arteries in patients with ST segment elevation myocardial infarctions (STEMIs). One prior study found that the FFRs of STEMI patients undergoing primary PCI were higher than those of angina pectoris patients with the same extents of stenosis [27].

However, in patients with non-ST segment elevation myocardial infarctions (NSTEMIs), the extents of microvascular dysfunction and myocardial stunning may be less than in those with STEMIs. The FAMOUS-NSTEMI (fractional flow reserve versus angiography in guiding management to optimize outcomes in non-ST elevation myocardial infarction) trial assessed the outcomes of NSTEMI patients undergoing routine FFR-guided or angiography-guided management [28]. In the FFR-guided group, the proportion of patients initially treated medically was higher than that in the angiography-guided group (22.7\% vs. $13.2 \%, p=0.022)$ and the extent of revascularization at 12 months was lower (79.0\% vs. 86.8\%, $p=0.054)$, but no significant between-group difference in terms of either overall health outcomes or the qual- 
ity-of-life was apparent. Although our present analysis supports the idea that FFR can be used to identify culprit lesions, the clinical events were too few in number to allow us to draw firm conclusions. Large-scale randomized trials with long-term follow-up are needed to assess the utility of FFR-guided management strategies in NSTEMI patients.

Several trials have focused on validation, and the clinical implications, of FFR guidance when non-infarct-associated arteries are to be treated in patients with MIs. Ntalianis et al. [29] measured FFR changes in nonculprit coronary artery lesions during the acute MI phase, and days and weeks later. The FFRs of nonculprit lesions did not change from the acute phase of MI to the 1-month follow-up (0.77 \pm 0.13 vs. $0.77 \pm 0.13, p=$ not significant). Therefore, FFR assessment of nonculprit lesions during the acute phase reliably assesses functional significance and usefully contributes to risk stratification. FFR-guided management of nonculprit lesions in patients with STEMIs was evaluated in the DANAMI-3-PRIMULTI (Primary PCI in Patients With ST-elevation Myocardial Infarction and Multivessel Disease: Treatment of Culprit Lesion Only or Complete Revascularization) trial, which only compared the clinical outcomes of patients who underwent FFR-guided CR during index admission in terms of revascularization of infarct-related arteries (IRAs) [30]. During 27 months of follow-up, the primary endpoints (death, MI, or ischemia-driven revascularization of non-IRAs) were attained less frequently in patients who underwent FFR-guided CR (13\% vs. $22 \%, p=$ 0.004). The Compare-Acute trial randomized FFR-guided treatment of non-IRAs in an acute setting. Non-IRA revascularization was performed only after successful primary PCI of IRA [31]. During the 12 month follow-up, the primary endpoint (a composite of death, MI, revascularization, and cerebrovascular events) was attained by $7.8 \%$ of the FFR-guided CR group and $20.5 \%$ of the IRA revascularization-only group $(p<0.001)$. Although recent evidence has suggested that the FFRs of acute myocardial infarction (AMI) patients with MVD is of prognostic significance, any role for this parameter should be further evaluated in the FRAME-AMI study (FFR versus Angiography-Guided Strategy for Management of AMI with Multivessel Disease; ClinicalTrials.gov No. NCT02715518).

\section{CONCLUSIONS}

Physiology-guided PCI based on the FFR in patients with MVD provided better clinical outcomes with lesser use of resources than angiography-guided PCI, regardless of the clinical presentation or disease subset. Selective revascularization of functionally significant lesions and a functional complete revascularization strategy assessed by FFR will allow for improved long-term outcomes.

\section{Conflict of interest}

No potential conflict of interest relevant to this article was reported.

\section{REFERENCES}

1. Mabin TA, Holmes DR Jr, Smith HC, et al. Follow-up clinical results in patients undergoing percutaneous transluminal coronary angioplasty. Circulation 1985;71:754-76o.

2. Corpus RA, House JA, Marso SP, et al. Multivessel percutaneous coronary intervention in patients with multivessel disease and acute myocardial infarction. Am Heart J 2004;148:493-500.

3. Min JK, Shaw LJ, Devereux RB, et al. Prognostic value of multidetector coronary computed tomographic angiography for prediction of all-cause mortality. J Am Coll Cardiol 2007;50:1161-1170.

4. Tonino PA, Fearon WF, De Bruyne B, et al. Angiographic versus functional severity of coronary artery stenoses in the FAME study fractional flow reserve versus angiography in multivessel evaluation. J Am Coll Cardiol 2010;55:2816-2821.

5. Levine GN, Bates ER, Blankenship JC, et al. 2011 ACCF/ AHA/SCAI guideline for percutaneous coronary intervention. A report of the American College of Cardiology Foundation/American Heart Association Task Force on Practice Guidelines and the Society for Cardiovascular Angiography and Interventions. J Am Coll Cardiol 2011;58:e44-e122.

6. Authors/Task Force members, Windecker S, Kolh P, et al. 2014 ESC/EACTS guidelines on myocardial revascularization: the task force on myocardial revascularization of the European Society of Cardiology (ESC) and the European Association for Cardio-Thoracic Surgery (EACTS) devel- 
oped with the special contribution of the European Association of Percutaneous Cardiovascular Interventions (EAPCI). Eur Heart J 2014;35:2541-2619.

7. Bypass Angioplasty Revascularization Investigation (BARI) Investigators. Comparison of coronary bypass surgery with angioplasty in patients with multivessel disease. $\mathrm{N}$ Engl J Med 1996;335:217-225.

8. BARI Investigators. The final 10-year follow-up results from the BARI randomized trial. J Am Coll Cardiol 2007;49:1600-1606.

9. Serruys PW, Unger F, Sousa JE, et al. Comparison of coronary-artery bypass surgery and stenting for the treatment of multivessel disease. N Engl J Med 2001;344:1117-1124.

10. Serruys PW, Ong AT, van Herwerden LA, et al. Five-year outcomes after coronary stenting versus bypass surgery for the treatment of multivessel disease: the final analysis of the Arterial Revascularization Therapies Study (ARTS) randomized trial. J Am Coll Cardiol 2005;46:575-581.

11. Serruys PW, Morice MC, Kappetein AP, et al. Percutaneous coronary intervention versus coronary-artery bypass grafting for severe coronary artery disease. N Engl J Med 2009;360:961-972.

12. Head SJ, Davierwala PM, Serruys PW, et al. Coronary artery bypass grafting vs. percutaneous coronary intervention for patients with three-vessel disease: final five-year follow-up of the SYNTAX trial. Eur Heart J 2014;35:2821-2830.

13. Park SJ, Ahn JM, Kim YH, et al. Trial of everolimus-eluting stents or bypass surgery for coronary disease. N Engl J Med 2015;372:1204-1212.

14. Gersh BJ, Frye RL. Methods of coronary revascularization: things may not be as they seem. N Engl J Med 2005;352:2235-2237.

15. Cho YK, Nam CW, Han JK, et al. Usefulness of combined intravascular ultrasound parameters to predict functional significance of coronary artery stenosis and determinants of mismatch. EuroIntervention 2015;11:163-170.

16. Melikian N, De Bondt P, Tonino P, et al. Fractional flow reserve and myocardial perfusion imaging in patients with angiographic multivessel coronary artery disease. JACC Cardiovasc Interv 2010;3:307-314.

17. Tonino PA, De Bruyne B, Pijls NH, et al. Fractional flow reserve versus angiography for guiding percutaneous coronary intervention. N Engl J Med 2009;360:213-224.

18. Pijls NH, Fearon WF, Tonino PA, et al. Fractional flow reserve versus angiography for guiding percutaneous coronary intervention in patients with multivessel coronary artery disease: 2-year follow-up of the FAME (Fractional Flow Reserve Versus Angiography for Multivessel Evaluation) study. J Am Coll Cardiol 2010;56:177-184.

19. van Nunen LX, Zimmermann FM, Tonino PA, et al. Fractional flow reserve versus angiography for guidance of PCI in patients with multivessel coronary artery disease (FAME): 5-year follow-up of a randomised controlled trial. Lancet 2015;386:1853-1860.

20. Fearon WF, Bornschein B, Tonino PA, et al. Economic evaluation of fractional flow reserve-guided percutaneous coronary intervention in patients with multivessel disease. Circulation 2010;122:2545-2550.

21. Nam CW, Mangiacapra F, Entjes R, et al. Functional SYNTAX score for risk assessment in multivessel coronary artery disease. J Am Coll Cardiol 2011;58:1211-1218.

22. Nam CW, Fearon WF. Role of the functional SYNTAX score in evaluating multivessel coronary artery disease. Interv Cardiol 2011;3:695-704.

23. Farooq V, Serruys PW, Bourantas CV, et al. Quantification of incomplete revascularization and its association with five-year mortality in the synergy between percutaneous coronary intervention with taxus and cardiac surgery (SYNTAX) trial validation of the residual SYNTAX score. Circulation 2013;128:141-151.

24. Choi KH, Lee JM, Koo B-K, et al. TCT-333 Prognostic implication of functional incomplete revascularization and residual functional SYNTAX score in patients with coronary artery disease. J Am Coll Cardiol 2017;70(18 Suppl):B137.

25. Ahn JM, Park DW, Lee CW, et al. Comparison of stenting versus bypass surgery according to the completeness of revascularization in severe coronary artery disease: patient-level pooled analysis of the SYNTAX, PRECOMBAT, and BEST trials. JACC Cardiovasc Interv 2017;10:1415-1424.

26. Kobayashi Y, Nam CW, Tonino PA, et al. The prognostic value of residual coronary stenoses after functionally complete revascularization. J Am Coll Cardiol 2016;67:1701-1711.

27. Tamita K, Akasaka T, Takagi T, et al. Effects of microvascular dysfunction on myocardial fractional flow reserve after percutaneous coronary intervention in patients with acute myocardial infarction. Catheter Cardiovasc Interv 2002;57:452-459.

28. Layland J, Oldroyd KG, Curzen N, et al. Fractional flow reserve vs. angiography in guiding management to optimize outcomes in non-ST-segment elevation myocardial in- 
farction: the British Heart Foundation FAMOUS-NSTEMI randomized trial. Eur Heart J 2015;36:100-111.

29. Ntalianis A, Sels JW, Davidavicius G, et al. Fractional flow reserve for the assessment of nonculprit coronary artery stenosis in patients with acute myocardial infarction. JACC Cardiovasc Interv 2010;3:1274-1281.

30. Engstrom T, Kelbaek H, Helqvist S, et al. Complete revascularisation versus treatment of the culprit lesion only in patients with ST-segment elevation myocardial infarction and multivessel disease (DANAMI-3-PRIMULTI): an open-label, randomized controlled trial. Lancet 2015;386:665-671.

31. Smits PC, Abdel-Wahab M, Neumann FJ, et al. Fractional flow reserve-guided multivessel angioplasty in myocardial infarction. N Engl J Med 2017;376:1234-1244. 